\title{
Conceptual processing is referenced to the experienced location of the self,
}

not to the location of the physical body

Elisa Canzoneri ${ }^{1}$, Giuseppe di Pellegrino ${ }^{2}$, Bruno Herbelin $^{1}$, Olaf Blanke $^{1}$, Andrea Serino ${ }^{1} *$

${ }^{1}$ Laboratory of Cognitive Neuroscience, Centre for Neuroprosthetics, École Polytechnique Fédérale de Lausanne, 1015 Lausanne, Switzerland; ${ }^{2}$ Department of Psychology, Alma Mater Studiorum, University of Bologna, 40100, Bologna, Italy.

* OB and AS equally contributed to the paper. 


\begin{abstract}
In this study we tested whether altering the experience of the body by means of Full Body Illusion (FBI) would impact high-level cognitive processing.

In an implicit association task, participants saw a landscape where words, conveying spatial ("Near", "Far") or social (“Us", "Them") semantic distance appeared either in a proximal or distal position. Words" "semantic" distance was congruent or incongruent to the words' spatial position. Participants were asked to classify words' spatial position. Implicit association judgements were intermingled with sessions of visuo-tactile FBI stimulation, during which participants were touched on their back, while viewing an avatar in the distal position being touched either synchronously or asynchronously. In a control experiment, participants performed the same task while observing a neutral object being touched, as a control.

When subjects self-identified with the avatar presented at the distal position, the facilitation effect of the words' spatial-semantic congruency disappeared. The congruency effect did not disappear in case of asynchronous stimulation as well as in the control experiment with the object, where no change in self-identification was observed. These results demonstrate that conceptual processing is not referenced to the location of the physical body, but to the location of the experienced self.
\end{abstract}

\title{
Keywords
}

Bodily Self-Counsciousness; Psychological Distance; Self; Multisensory processing; Embodied cognition 


\section{Introduction}

Theories of embodied cognition (Barsalou, 2008; Gibbs, 2006; Gallagher, 2005; Borghi \& Cimatti, 2010) suggest the importance of sensory-motor systems and the body for cognition, by posing that our mental processes, even high-level cognitive processes, are situated in and depend on the physical body. In line with this view the Construal Level Theory (CLT) of psychological distance by Lieberman \& Trope (Liberman \& Trope, 2008, 2014; Trope \& Liberman, 2010) posits that different aspects of semantic information processing, i.e., spatial, temporal, social and probabilistic reasoning, are processed as referenced to the body. More specifically, these concepts are represented on a common dimension in terms of psychological distance from one's own physical body (Bar-Anan, Liberman, Trope, \& Algom, 2007; Liberman \& Trope, 2008; 2014). Typical empirical demonstrations of this proposal come from a series of experiments (see e.g., Bar-Anan et al., 2007 and Trope \& Liberman, 2010 for a review), whereby participants underwent an implicit association task during which they were presented with pictures containing spatial depth cues, and words denoting either psychological proximity ("Near", "Now", "Us", "Sure") or psychological remoteness meaning ("Far", "Tomorrow", "Them", "Maybe") in different semantic domains. Words were placed either close to the participant's point of view, or far in the scene. The participant's task was to indicate as quickly as possible whether the word was located spatially near or far in the scene. Typically, the pattern of responses revealed an interference effect: participants respond faster to words whose spatial location and psychological distance matched (e.g. the word "Near" placed at the proximal spatial location on the scene, congruent condition), as compared to not matching stimuli (e.g., the word "Near" placed at the distal spatial location on the scene, incongruent condition). This effect has been 
interpreted as a demonstration that words' meaning for different conceptual domains are automatically and implicitly processed in terms of spatial distance from one's body (termed "psychological distance"). We generally do not discriminate between spatial distance from the self and spatial distance from the physical body, likely because normally the experience of our self is bounded at that of the physical body: we perceive our self at the place occupied by the body. Such link between the self and the body is a central feature of self-consciousness and is captured by the concept of bodily self-consciousness (BSC; Blanke \& Metzinger, 2009; Vogeley \& Fink, 2003; Blanke, 2012; Pfeiffer, Lopez, Schmutz, Duenas, Martuzzi, \& Blanke, 2013). It has been proposed that BSC arises from multisensory integration of body-related signals (Blanke, 2012), suggesting a close link between body-related information processing and self experience.

As discussed above, although self-experience normally coincides with the location of one's own body in spatial terms, conflicting multisensory stimuli can temporarily alter this spatial unity between body and self. During the so-called Full Body Illusion (FBI; Lenggenhager, Tadi, Metzinger, \& Blanke, 2007; Ehrsson, 2007; Aspell, Lavanchy, Lenggenhager, \& Blanke, 2010; see also Petkova \& Ehrsson, 2008), participants view the video image of their body filmed from behind and projected in front of them, while an experimenter concurrently strokes their back. Viewing and feeling one's own back being stroked synchronously - but not asynchronously - induces changes in BSC, leading to a drift in self-location towards the seen (distant) body and self-identification with the seen body. Such a change does not apply when participants view a neutral object being stroked, instead of their own body.

Such changes in BSC have been shown to be associated with changes in low-level, 
body related, information processing, such as changes in tactile responses (Aspell, Lenggenhager, \& Blanke, 2009), skin conductance responses (Ehrsson, 2007; Petkova \& Ehrsson, 2008), pain perception (Hänsel, Lenggenhager, von Känel, Curatolo, \& Blanke, 2011; Romano, Pfeiffer, Maravita, \& Blanke, 2014) and body temperature (Moseley, Olthof, Venema, Don, Wijers, Gallace, \& Spence, 2008; Salomon, Lim, Pfeiffer, Gassert, \& Blanke, 2013).

Moreover, previous studies in the literature have also demonstrated a link between the sense of bodily self - as manipulated by means of classical body illusions - and highlevel information processing. For instance, it has been demonstrated that illusory ownership of a dark-skinned rubber hand (Maister, Sebanz, Knoblich, \& Tsakiris, 2013) or virtual body (Peck, Seinfeld, Aglioti, \& Slater, 2013) reduces implicit racial bias and changes implicit social attitude towards outgroups. Banakou and colleagues (Banakou, Groten, \& Slater, 2013) showed how the illusory ownership of a virtual child body causes implicit perceptual changes, while the illusory ownership of an invisible body reduces social anxiety responses (Guterstam, Abdulkarim, \& Ehrsson, 2015). The general idea underlying these studies is that by changing the similarity between the self and the others through shared multisensory experiences can create a connection between the "low-level" representation of the body and high-level cognitive processing as for instance social cognition and social-affective processes. By using patterns of visuo-tactile or visuo-motor stimulation, these studies successfully induced embodiment for a "physically" different body or body part, while manipulating the color of skin of the virtual body part (i.e. the arm, Maister et al., 2013) or of the virtual full body (Peck et al., 2015; see also Maister, Slater, Sanchez-Vives \& Tsakiris, 2015 for a review) as well as the age, sex (Slater, Spanlang, Sanchez-Vives, \& Blanke, 2010), body dimension (Banakou et al., 2013) 
and also presence (embodiment of an invisible body, Guterstam et al., 2015) of the virtual body. Here instead we used the FBI to experimentally manipulate the unity between the physical body and the experienced self and test whether such alteration of BSC would in turn affect higher-level cognitive processes, of which we take semantic processing as a paradigmatic case. More specifically, we hypothesized that altering the perceived location of the self in space through the FBI, which affects the spatial coincidence between the self and the body, would change the reference point to which psychological distance evoked by words is computed. We were particularly interested in exploring the presence of such a link in the field of the CLT, since this theory provides a conceptualization of distance of different concepts as anchored to a point of egocentric reference that normally coincides with the body. Here, by manipulating the spatial unity between the physical body and the self by means of the FBI we asked whether such egocentric reference for psychological distance coincide with the former or the latter. To test this hypothesis, participants underwent an adapted version of the implicit association task by Bar-Anan and colleagues (Bar-Anan et al., 2007), while being exposed to visuo-tactile stimulation either inducing the FBI (synchronous condition) or not inducing the FBI (asynchronous control condition). Participants were presented with a landscape scene in a virtual environment. A word could appear superimposed on the image, either at a proximal or at a distal location, close to where an avatar body was shown (see Figure 1). Each word conveyed a concept, which was either congruent or incongruent in terms of psychological distance meaning for spatial ("Near" vs. "Far"; Experiment 1), or social ("Us" vs. "Them"; Experiment 2), dimension with the location of the word on the scene with respect to the participants' point of view. Participants were asked to classify as quickly as possible the spatial position of the word in the scene, immediately following exposure to the FBI. Based 
on previous results by Bar-Anan and colleagues (2007), in the asynchronous condition we predicted participants to respond faster to words located at congruent psychological distances from their physical body. Psychological distance, indeed, would be computed as referenced to the location of the self, which, in the case of asynchronous stimulation, overlaps with the location of the physical body, matching the proximal position of the words on the screen. Conversely, when exposed to synchronous visuo-tactile stimulation inducing the FBI, the facilitation due to congruent words in terms of both spatial and social psychological distance should reduce or disappear, as the FBI produces an illusory effect of self-identification with the virtual body, which is placed at the distal location on the scene. This pattern of results would suggest that concepts conveying psychological distance are processed depending on the perceived location of the self in space, more than on the physical location of the body. Moreover, in order to demonstrate that the results at the implicit association task depend on a change in BSC, and were not an effect of shifting spatial attention towards a far location, in Experiment 3 participants performed the same experiment as Experiment 1, with the only difference that, instead observing the virtual body during visuo-tactile stimulation they observed a body-sized object of a rectangular shape. It is known that viewing an object being stroked does not induce the same changes in BSC induced by viewing a bodily stimulus (Blanke, Slater, \& Serino, 2015; Tsakiris, 2010); thus, such condition should not affect how participants process psychological distance related concepts. 


\section{Methods}

\subsection{Participants}

Thirty-six subjects participated in the experiment. Twelve subjects participated in Experiment 1 (seven males, mean age \pm SD, 22 \pm 2.9 ), twelve subjects participated in Experiment 2 (five males, 22 \pm 3 ) and the remaining twelve subjects (six males, 23 \pm 5 ) participated in Experiment 3. All subjects were native French speakers and they were reimbursed for their time. All subjects were naive to the purpose of these experiments and gave informed written consent. The experiment conformed to institutional guidelines and to the Declaration of Helsinki.

\subsection{Experiment 1 and 2}

\subsubsection{Materials}

Participants underwent an implicit association task similar to the one of BarAnan and colleagues (2007), adapted to a virtual reality environment. Participants were presented with a landscape scene through a Head Mounted Display (HMD VR1280 Virtual Research Systems, Inc.; Dual SXGA 1280 x 1024 resolution, 60 diagonal field of view). The scene was selected among others landscape scenes on the Internet through Google's Images search tool. The image was chosen in order to convey a clear depth perception and previously tested in a pilot study, so to be sure that participants would be able to easily report the spatial location of an object (near or far) on the picture. A target stimulus (a word printed on a green arrow) appeared superimposed in each of these images. In different trials, the green arrow could be presented slightly on the left or on the right side of the image, either in a spatially proximal or a spatially distal position. To overcome for perspectival cues, the font 
size of the words was 24 when they were printed on a spatially proximal arrow and 18 when they were printed on a spatially distal arrow (as in Bar-Anan et al., 2007; See Figure 1 for an illustration of the makeup of the stimuli).

The implicit association task was combined with the visuo-tactile stimulation used to manipulate BSC. During the entire experiment participants saw the back of their bodies filmed from a distance of $3 \mathrm{~m}$ and projected onto the virtual scenario as superimposed in the scene, in a position corresponding to where the distal words were presented during the implicit association task.

All participants wore a white t-shirt and they were standing in the middle of a room, with their finger on a keyboard placed on a table in front of them. During visuo-tactile stimulation, the back (the area spanning the shoulders to waist) of participants was irregularly stroked - about twice per second - by the experimenter with a long wooden stick, and participants could see the visuo-tactile stimulation on the virtual scene via the HMD. In asynchronous blocks, a delay of $500 \mathrm{~ms}$ was introduced so that "seen" stimulation and "felt" stimulation did not temporally correspond. Participants were instructed to fixate the middle of their virtual back during the visuo-tactile stimulation. To ensure that participants were paying attention to visual stimulation on the virtual body, a red dot, flashing for $500 \mathrm{~ms}$, was randomly presented on participants' back during the visuo-tactile stimulation for 6 times for each experimental session. Participants were asked to count them. At the end of each block participants were asked to report the total number of the red dots.

\subsubsection{Procedure}

At the beginning of the experiment, participants were briefed about the stimuli they were going to experience, and instructed on the task requests. Participants wore 
the HMD, and then the experiment adjusted the two cameras' behind participants' body, so that they could clearly see their back from behind, projected into the HMD. A training session was carried out before the beginning of the experiment, in which participant experienced the visuo-tactile stimulation and performed a trial version (20 trials) of the implicit association task. Each experimental block consisted of four subblocks of visual-tactile stimulation intermingled with four-sub blocks of the implicit association task. Each sub-block of visuo-tactile stimulation lasted one minute. In Experiment 1 participants were presented with pair of words conveying spatial distance ("Near", "Far"), while in Experiment 2 participants were presented with pair of words conveying social distance ("Us", "Them"). Participants also performed the task with control words ("Screw", "Nail”), not implying any psychological distance meaning, in a different block. Each word was presented in French that was the native language for all participants. Each of the 4 images (2 spatial locations X 2 words) was presented 8 times for each sub-block, thus creating a total of 128 stimuli per block. Participants performed two blocks for each pair of words, one block in the Synchronous visuo-tactile condition and one block in the Asynchronous visuo tactile condition, for a total of four blocks for participants (Psychological Distance words Synchronous and Psychological Distance words Asynchronous; Control words Synchronous and Control words Asynchronous). The order of blocks (Psychological Distance blocks and Control blocks) was counterbalanced across participants. The order of visuo-tactile stimulation (Asynchronous or Synchronous) was randomized for each participant.

For the implicit association task, participants responded using two different keys (M or $\mathrm{N}$ ) on a keyboard to indicate whether the target stimulus was presented near or far in the perspective of the image, by using the index and the middle finger of the 
dominant hand. Half participants performed the task pressing $M$ for the distal stimuli and $\mathrm{N}$ for the proximal stimuli, and vice-versa for the remaining participants.

After having completed each experimental block, participants completed a questionnaire pertaining to the visuo-tactile stimulation and developed to assess the FBI (adapted from Lenggenhager et al., 2007; see also Aspell, Heydrich, Marillier, Lavanchy, Herbelin, \& Blanke, 2013). They were presented with a series of four statements describing changes in BSC due visuo-tactile stimulation (e.g., "I felt as if the virtual body was my body"; Q1-Q4) and four control statements (Q5-Q8) and they were asked to rate their agreement on a visual analogue scale with scores ranging from -3 (absolutely disagree) to +3 (absolutely agree). Questions are reported in Figure 2.

a)

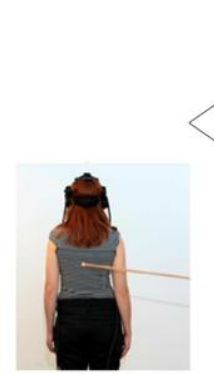

b)

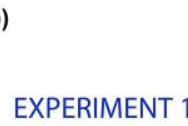

EXPERIMENT 2

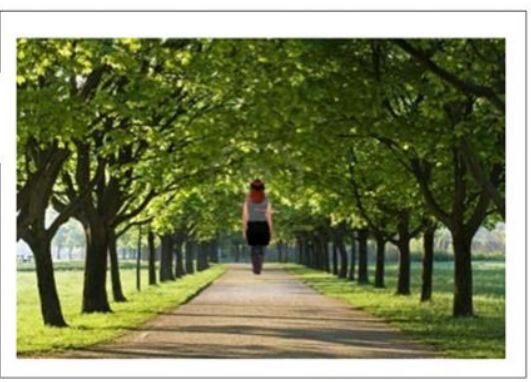

CONGRUENT INCONGRUENT

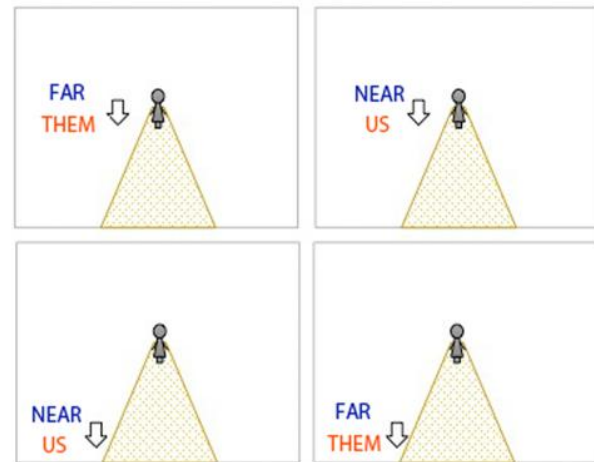

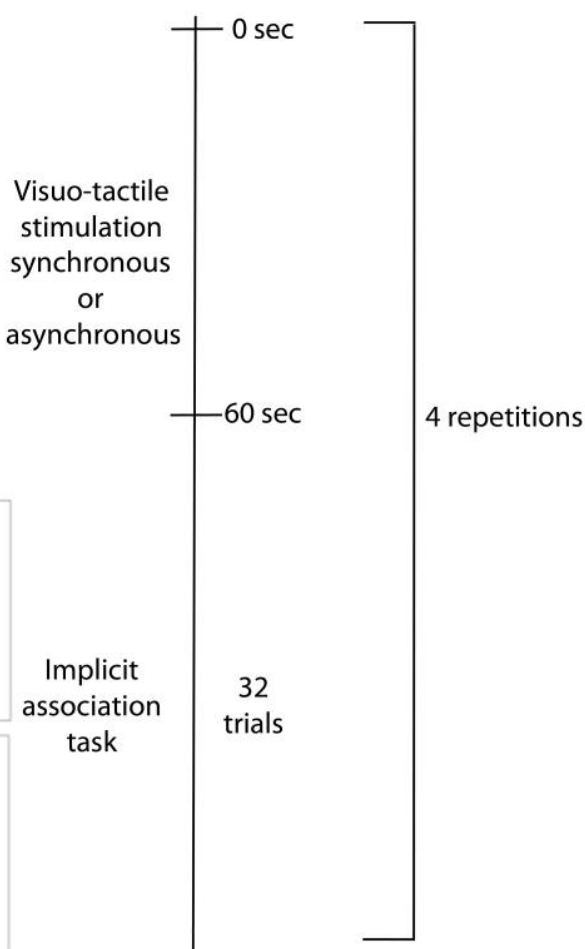

Questionnaire

Figure 1. Experimental set-up, makeup of the stimuli and procedure. The figure shows a 
representation of the stimuli (on the left) and a schematic representation of each block structure (on the right).

(a) Example of the visuo-tactile stimulation as seen from each subject through the HMD. (b) Schematic example of different trials combination for the implicit association task. Congruent conditions are represented by trials where the semantic meaning of the word matched the spatial location on the scene (i.e. the word "Far" for Experiment 1 and "Them" for Experiment 2 presented in a distal spatial location and the word "Near" for Experiment 1 and "Us" for Experiment 2 presented in a proximal spatial location). Incongruent conditions are represented by trials where the semantic meaning of the word did not match the spatial location on the scene (i.e. the word "Far" for Experiment 1 and "Them" for Experiment 2 presented in a proximal spatial location and the word "Near" for Experiment 1 and "Us" for Experiment 2 presented in a distal spatial location).

\subsection{Experiment 3}

\subsubsection{Materials}

The Experiment was exactly the same as Experiment 1, except for the visuo-tactile stimulation phase. Participants underwent the same tactile stimulation on their back as described above for the previous experiment, but in this case participants could see the visuo-tactile stimulation on the virtual scene via the HMD applied on a virtual object. The object was a rectangular-shaped box of approximately 120 by $50 \mathrm{~cm}$. Participants were instructed to fixate the middle of the virtual object during the visuotactile stimulation. To ensure that participants were paying attention to visual stimulation on the virtual object, a red dot, flashing for $500 \mathrm{~ms}$, was randomly presented on the object during the visuo-tactile stimulation for 6 times for each experimental session. Participants were asked to count them. At the end of each block participants were asked to report the total number of the red dots.

\subsubsection{Procedure}

The experimental procedure was exactly the same as for Experiment 1. Since it has been previously demonstrated that BSC is not modulated during the FBI while visuotactile stimulation is applied on a human-sized object (see Lenggenhager et al., 2007; Aspell et al., 2009), participants did not complete the FBI questionnaire at the end of 
this experiment.

\section{Data analysis}

\subsection{Subjective responses}

The data from each question underwent an intra-subject standardization by means of an ipsatization procedure in order to neutralize the effect in responses set (Broughton \& Wasel, 1990; Cattell, 1994). Specifically, each rating was subtracted by the mean rating of the subject responses in all questions and conditions and then divided by the standard deviation of subject's responses in all questions and conditions. Ipsatization transformed questionnaire ratings in Z-scores with a normal distribution allowing a proper use of parametric tests on questionnaire data (Broughton \& Wasel, 1990; Cattell, 1994; see also Romano et al., 2014).

\subsection{Implicit Association Task}

For the implicit association task, in line with previous reports (see Bar-Anan et al., 2007), the performance was analysed in terms of reaction time only. Mean reaction time (RTs) to the target words was calculated for each condition separately (see below). Trials where participants gave a wrong response were trimmed from the analysis $(6.1 \%$ of trials on average in all conditions). RTs exceeding more than 2 standard deviations from the mean RT were considered outliers and trimmed from the analysis (1.8\% of trials on average in all conditions).

\section{Experiment 1 and 2}

\section{Results}

\subsection{Subjective responses}

In order to analyse the subjective responses, we used a standard approach 
adopted in several studies using body illusions (Preston \& Ehrsson, 2014; Aspell et al., 2013; Slater, Perez-Marcos, Ehrsson, \& Sanchez-Vives, 2008). We averaged Illusion Questions (Q1-Q2-Q3-Q4) and Control Questions (Q5-Q6-Q7-Q8) for each subject and a repeated measure ANOVA was conducted on the ipsatized values with the factors Types of Questions (Experimental, Control), Stimulation (Synchronous, Asynchronous) and Word (Psychological Distance, Control) as within subject factors, and Experiment (Experiment 1, Experiment 2) as a between subject factor.

The ANOVA revealed that Experimental and Control Questions were modulated depending on the Stimulation, as expected, as revealed by the two-way interaction between Stimulation and Types of Questions $\left[F(1,22)=13.39, p<0.01, \eta^{2}=0.38\right]$. In order to explore this interaction, we then analysed Experimental and Control Questions separately with a repeated measure ANOVA with Stimulation (Synchronous, Asynchronous) and Words (Psychological Distance, Control) as within subject factors, and Experiment (Experiment 1, Experiment 2) as a between subject factor.

The ANOVA conducted on the Experimental Questions revealed a main effect of Stimulation $\left[\mathrm{F}(1,22)=5.27, \mathrm{p}<0.01, \eta^{2}=0.51\right]$. As expected, participants rated the experience of the illusion as stronger after Synchronous stimulation (Mean, 95\% confidence intervals or $\mathrm{CI}: \mathrm{Z}=0.51$, $\mathrm{CI}[0.38,0.65])$ as compared to Asynchronous stimulation $(Z=-0.17$, CI $[-0.38,0.04]$; see Figure 2$)$. No other effects were found. This result suggests that Synchronous visuo-tactile stimulation induced a change in BSC, such as participants identified with the virtual body when synchronous visuotactile stimulation was applied to the virtual body and to their real body. The strength of the illusion did not vary between the different blocks of the implicit association 
task and the different experiments, since there was no main effect of Words or Experiment, nor any interaction (all $\mathrm{p}_{\mathrm{s}}>0.32$ ). This differed for the Control Questions: the synchrony of visuo-tactile stimulation did not affect participants' ratings, as the main effect of Stimulation was not significant $\left(\left[\mathrm{F}(1,22)=0.05, \mathrm{p}=0.83, \eta^{2}=0.002\right.\right.$; Synchronous: $\mathrm{Z}=-0.16$, CI [-0.30, -0.02]; Asynchronous: $\mathrm{Z}=-0.18$, CI [-0.33, -0.03]). No other effects were found.

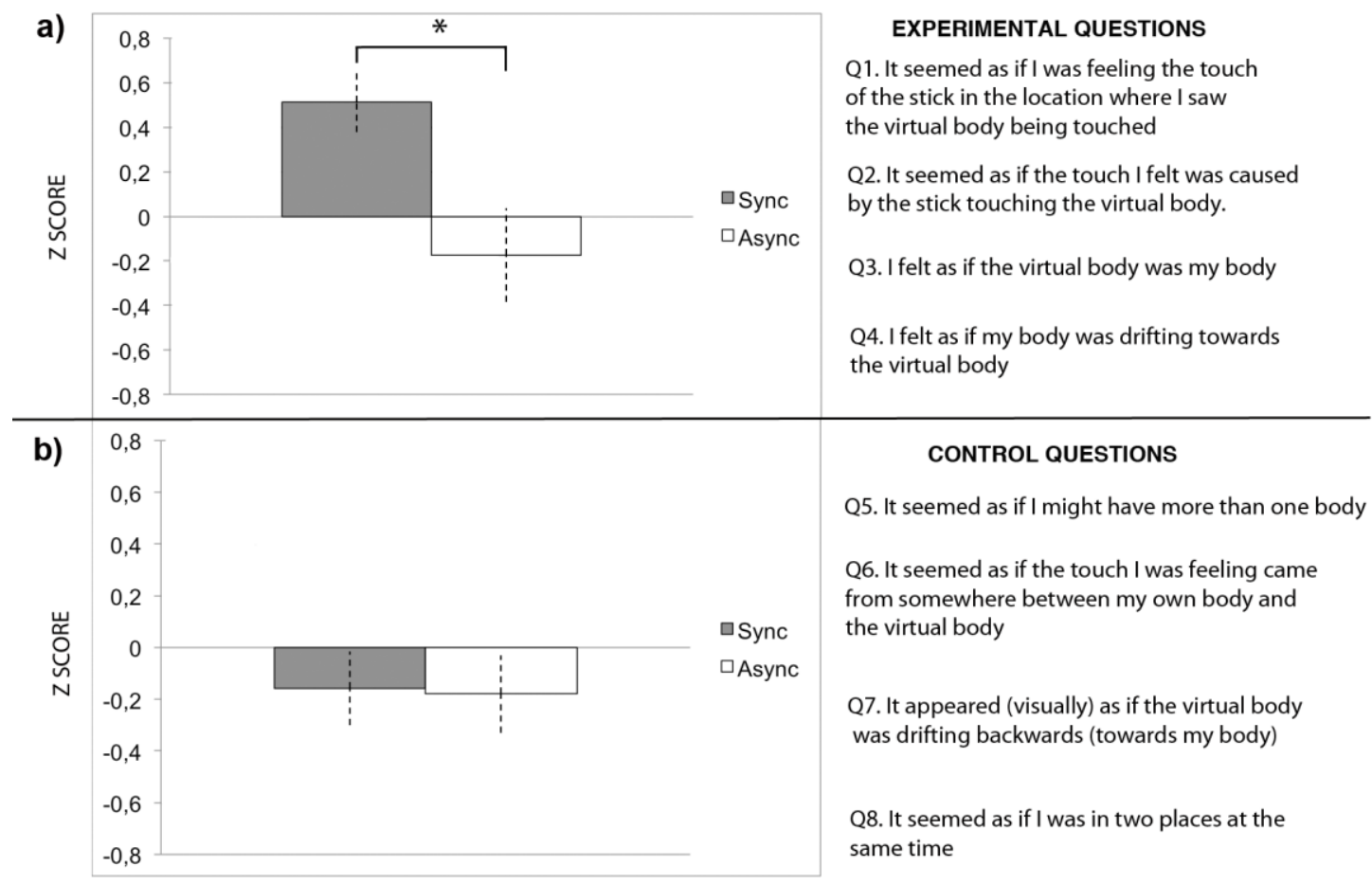

Figure 2. Subjective data. Mean questionnaire ratings, transformed in Z-scores, for Synchronous and Asynchronous condition, for Experimental questions (average of Q1-Q4, Panel A) and Control questions (average of Q5-Q8, Panel B). Error bars represent 95\% confidence intervals. Full results are summarized in Table 1. 


\begin{tabular}{|c|c|c|c|c|c|c|c|c|}
\hline \multirow{2}{*}{ Experiment 1 } & \multicolumn{7}{|c|}{ Synchronous } \\
\cline { 2 - 9 } & Q1 & Q2 & Q3 & Q4 & Q5 & Q6 & Q7 & Q8 \\
\hline $\begin{array}{c}\text { Psychological } \\
\text { Distance }\end{array}$ & $\begin{array}{c}2 \\
{[-1,3]}\end{array}$ & $\begin{array}{c}-1 \\
{[-3,3]}\end{array}$ & $\begin{array}{c}0 \\
{[-3,3]}\end{array}$ & $\begin{array}{c}-2 \\
{[-3,3]}\end{array}$ & $\begin{array}{c}-2 \\
{[-3,3]}\end{array}$ & $\begin{array}{c}0 \\
{[-3,2]}\end{array}$ & $\begin{array}{c}-2 \\
{[-3,2]}\end{array}$ & $\begin{array}{c}-2 \\
{[-3,2]}\end{array}$ \\
\hline \multirow{2}{*}{ Control } & $\begin{array}{c}2 \\
{[-1,3]}\end{array}$ & $\begin{array}{c}0 \\
{[-2,3]}\end{array}$ & $\begin{array}{c}0 \\
{[-3,3]}\end{array}$ & $\begin{array}{c}-2 \\
{[-3,2]}\end{array}$ & $\begin{array}{c}-2 \\
{[-3,2]}\end{array}$ & $\begin{array}{c}-2 \\
{[-3,1]}\end{array}$ & $\begin{array}{c}-2 \\
{[-3,2]}\end{array}$ & $\begin{array}{c}0 \\
{[-3,2]}\end{array}$ \\
\hline \multirow{2}{*}{$\begin{array}{c}\text { Psychological } \\
\text { Distance }\end{array}$} & $\begin{array}{c}-1 \\
{[-3,3]}\end{array}$ & $\begin{array}{c}-2 \\
{[-3,2]}\end{array}$ & $\begin{array}{c}-2 \\
{[-2,3]}\end{array}$ & $\begin{array}{c}-2 \\
{[-3,2]}\end{array}$ & $\begin{array}{c}-1 \\
{[-3,2]}\end{array}$ & $\begin{array}{c}-1 \\
{[-3,3]}\end{array}$ & $\begin{array}{c}-1 \\
{[-3,2]}\end{array}$ & $\begin{array}{c}-1 \\
{[-3,2]}\end{array}$ \\
\hline & $\mathbf{Q 1}$ & $\mathbf{Q 2}$ & $\mathbf{Q 3}$ & $\mathbf{Q 4}$ & $\mathbf{Q 5}$ & $\mathbf{Q 6}$ & $\mathbf{Q 7}$ & $\mathbf{Q 8}$ \\
\hline Control & $\begin{array}{c}-2 \\
{[-3,3]}\end{array}$ & $\begin{array}{c}-2 \\
{[-3,3]}\end{array}$ & $\begin{array}{c}-1 \\
{[-3,3]}\end{array}$ & $\begin{array}{c}-2 \\
{[-3,3]}\end{array}$ & $\begin{array}{c}-2 \\
{[-3,1]}\end{array}$ & $\begin{array}{c}-2 \\
{[-3,1]}\end{array}$ & $\begin{array}{c}-3 \\
{[-3,3]}\end{array}$ & $\begin{array}{c}-2 \\
{[-3,2]}\end{array}$ \\
\hline
\end{tabular}

\begin{tabular}{|c|c|c|c|c|c|c|c|c|}
\hline \multirow{2}{*}{ Experiment 2 } & \multicolumn{7}{|c|}{ Synchronous } \\
\cline { 2 - 9 } & Q1 & Q2 & Q3 & Q4 & Q5 & Q6 & Q7 & Q8 \\
\hline $\begin{array}{c}\text { Psychological } \\
\text { Distance }\end{array}$ & $\begin{array}{c}2 \\
{[-3,3]}\end{array}$ & $\begin{array}{c}2 \\
{[-3,3]}\end{array}$ & $\begin{array}{c}1 \\
{[-3,3]}\end{array}$ & $\begin{array}{c}-2 \\
{[-3,3]}\end{array}$ & $\begin{array}{c}0 \\
{[-3,2]}\end{array}$ & $\begin{array}{c}-1 \\
{[-3,2]}\end{array}$ & $\begin{array}{c}-2 \\
{[-3,2]}\end{array}$ & $\begin{array}{c}-1 \\
{[-3,2]}\end{array}$ \\
\hline \multirow{2}{*}{ Control } & $\begin{array}{c}2 \\
{[-1,3]}\end{array}$ & $\begin{array}{c}2 \\
{[-2,3]}\end{array}$ & $\begin{array}{c}1 \\
{[-3,2]}\end{array}$ & 0 & -1 & -1 & -1 & 1 \\
{$[-3,2]$} & {$[-3,2]$} & {$[-3,1]$} & {$[-3,1]$} & {$[-3,3]$} \\
\hline \multirow{2}{*}{$\begin{array}{c}\text { Psychological } \\
\text { Distance }\end{array}$} & $\begin{array}{c}-2 \\
{[-3,3]}\end{array}$ & $\begin{array}{c}-2 \\
{[-3,2]}\end{array}$ & $\begin{array}{c}-1 \\
{[-3,2]}\end{array}$ & $\begin{array}{c}-2 \\
{[-3,1]}\end{array}$ & $\begin{array}{c}-2 \\
{[-3,2]}\end{array}$ & $\begin{array}{c}-1 \\
{[-3,2]}\end{array}$ & $\begin{array}{c}-1 \\
{[-3,3]}\end{array}$ & $\begin{array}{c}-1 \\
{[-3,2]}\end{array}$ \\
\hline & $\mathbf{Q 1}$ & $\mathbf{Q 2}$ & $\mathbf{Q 3}$ & $\mathbf{Q 4}$ & $\mathbf{Q 5}$ & $\mathbf{Q 6}$ & $\mathbf{Q 7}$ & $\mathbf{Q 8}$ \\
\hline Control & -2 & -1 & 0 & -1 & 1 & -1 & -1 & 1 \\
{$[-3,3]$} & {$[-32]$} & {$[-2,2]$} & {$[-3,1]$} & {$[-3,2]$} & {$[-3,3]$} & {$[-3,2]$} & {$[-3,2]$} \\
\hline
\end{tabular}

Table 1. Full raw results of the Questionnaire (expressed as median and interquartile ranges), for Experiment 1 (upper part) and Experiment 2 (lower part), separately for Psychological Distance and Control blocks, both for Synchronous and Asynchronous stimulation. 


\subsection{Implicit association task}

RTs for Psychological Distance blocks were compared by means of a repeated measure ANOVA with Stimulation (Synchronous, Asynchronous) and Congruency (Congruent, Incongruent) as within-subjects factors. Since in the context of the CLT spatial and social distances are considered as evoking the same psychological distance effect (Liberman \& Trope, 2014), results from Experiment 1 (with words expressing spatial distance) and Experiment 2 (with words expressing social distance) were analysed in the same ANOVA, by adding the factor Experiment as a between subject factor. RTs from blocks with control words were instead analysed by means of a separate repeated measure ANOVA with Stimulation (Synchronous, Asynchronous) and Words ("Screw", "Nail") as within subject factors and Experiment (Experiment 1, Experiment 2) as between-subjects factor.

For Psychological Distance blocks, analysis showed that the classical congruency effect was modulated by the nature of visuo-tactile stimulation, as revealed by the Stimulation $x$ Congruency interaction $\left[F(1,22)=16.16, p<0.001, \eta^{2}\right.$ $=0.42]$. Newman-Keuls post hoc tests showed that - as predicted - in case of Asynchronous visuo-tactile stimulation, participants were faster with congruent trials (Mean, 95\% confidence intervals or CI: $502 \mathrm{~ms}, 95 \% \mathrm{CI}=[467,536]$ as compared to incongruent trials $(525 \mathrm{~ms}, 95 \% \mathrm{CI}=[492,557] \mathrm{p}<0.001)$. This shows that participants responded as reported previously in the literature: they responded faster to the location of the arrow on the screen when the superimposed words evoked a semantic meaning matching, in terms of psychological distance, the spatial distance from their physical body (or the self, as experienced at the location of the physical 
body; see Figure 3A; see also Bar-Anan et al., 2007). This was different after Synchronous visuo-tactile stimulation: the difference between congruent and incongruent trials was not present anymore (Congruent: $508 \mathrm{~ms}, 95 \% \mathrm{CI}=[479$, 538]; Incongruent: $506 \mathrm{~ms}, 95 \% \mathrm{CI}=[479,532], \mathrm{p}=0.62)$. The pattern of results was the same for words conveying spatial (Experiment 1) and social (Experiment 2) distance, since the 3-way interaction with the factor Experiment was not significant $[F(1,22)=0.004, p=0.95)$. Importantly, for the Control blocks, RTs were neither affected by Stimulation nor Words, since no main effect of Stimulation $[F(1,22)=0.56$, $\left.\mathrm{p}=0.46, \eta^{2}=0.03\right]$ or Words $\left[\mathrm{F}(1,22)=82, \mathrm{p}=0.37, \eta^{2}=0.04\right]$, nor the two-way interaction were significant $\left.\left[\mathrm{F}(1,22)=0.34, \mathrm{p}=0.56, \eta^{2}=0.02\right]\right)$. As expected, there was no interaction with the factor Experiment (all $\left.\mathrm{p}_{\mathrm{s}}>0.26\right)$ as well. 

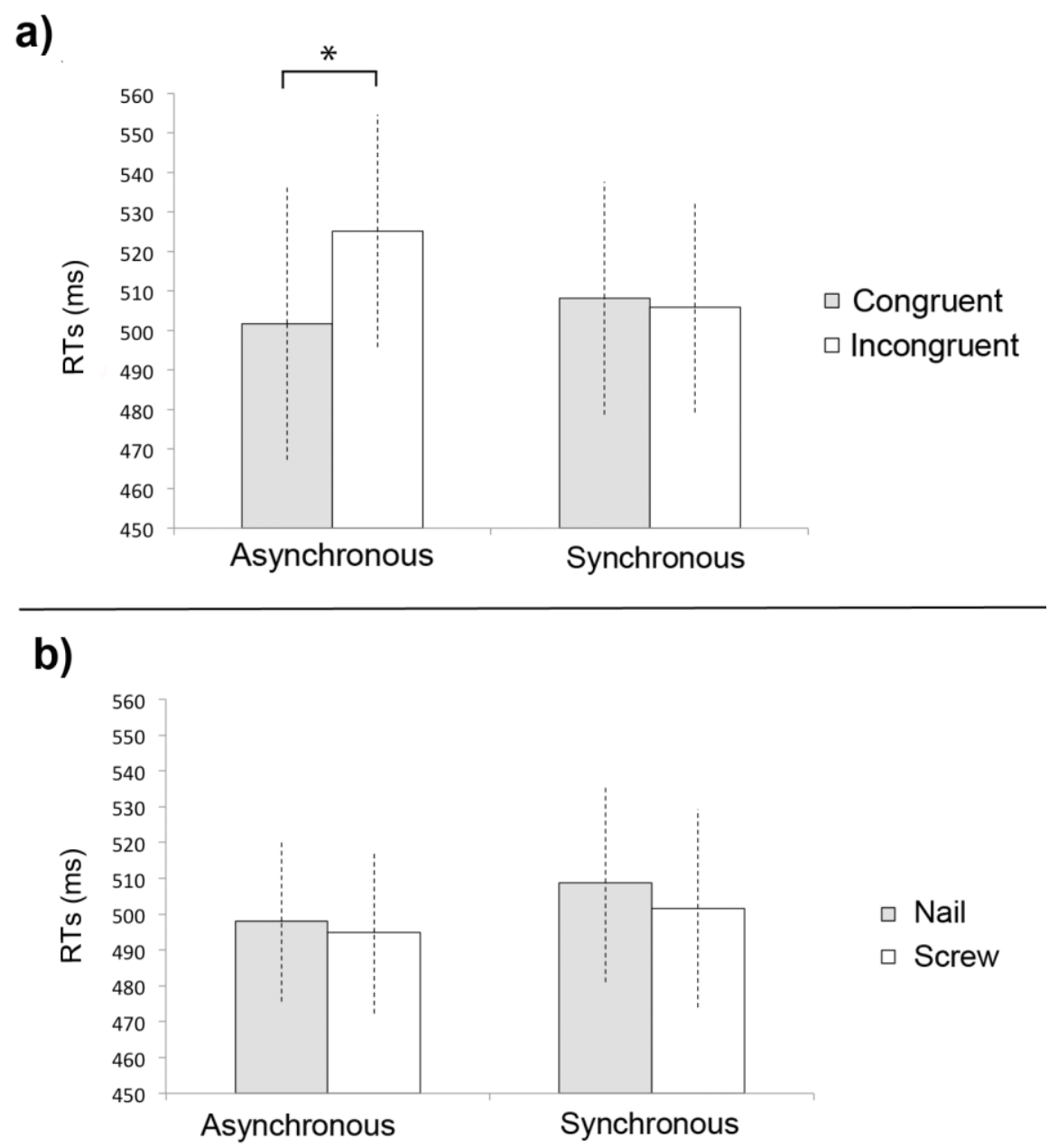

Figure 3. Implicit Association Task results. Mean RTs for target words shown separately for Psychological Distance (Panel A) and Control (Panel B) blocks.

Panel A. Mean RTs for Congruent and Incongruent conditions both for Synchronous and Asynchronous stimulation. Congruent condition refers to trials where spatial location matched the psychological distance of the words (e.g. the word "Us/Near" presented at the proximal location in the image), while Incongruent refers to trials where spatial location and psychological distance of the words did not match (e.g. the word "Us/Near" presented at the distal position in the image).

Panel B. Mean RTs for control Words (Screw, Nail) both for Synchronous and Asynchronous stimulation.

We then ran a second analysis on Psychological Distance blocks' data, to further understand how the change in BSC induced by synchronous visuo-tactile stimulation 
abolished the well-established psychological distance effect. To this aim, we further analysed RTs by taking into account the spatial location of the word in the scene as a factor in the analysis. We conducted a repeated measure ANOVA with Stimulation (Synchronous, Asynchronous), Spatial Location (Far, Near), and Word Semantic Distance (Proximal, Distal) as within subject factors, and Experiment (Experiment 1: Spatial distance; Experiment 2: Social distance) as between subject factor. Proximal words were "Near" and "Us" respectively for Experiment 1 and 2, while Distal words were "Far" and "Them", respectively for Experiment 1 and 2. The distal position in the scene corresponded to the location the participants identified with in the Synchronous condition (see Questionnaire results). Thus, we predicted that RTs to words located at that distal position would be more specifically affected by synchronous visuo-tactile stimulation. The three way Stimulation X Spatial Location $\mathrm{X}$ Words Semantic Distance interaction was significant $[\mathrm{F}(1,22)=16.16 \mathrm{p}<0.001, \eta$ $\left.{ }^{2}=0.30\right]$. As shown in Figure 4, Newman-Keuls post-hoc comparisons showed that after Synchronous visuo-tactile stimulation, the pattern of results differed between distal and proximal locations. Specifically, for words presented in the distal position of the scene, participants become faster in responding to stimuli expressing incongruent (that is, Proximal Words) spatial-semantic "psychological distance" compared to stimuli expressing congruent (Distal Words) spatial-semantic "psychological distance" (Proximal Words $=508 \mathrm{~ms}, 95 \% \mathrm{CI}=[479,536]$, Distal Words $=527 \mathrm{~ms}, 95 \% \mathrm{CI}=[496,557], \mathrm{p}<0.03)$. Put in another way, faster RTs were now recorded when a word expressing either spatial (Experiment 1) or social (Experiment 2) proximity was presented in the distal position in the image, that is at the position of the avatar that our participants identified with. This result suggests that, after a change in BSC - whereby participants self identified with the virtual body that 
was seen in the distal position of the scene - the words' semantic distance was not automatically referenced to the location of the participant's physical body anymore, but to the position of the virtual body. For the words presented in the proximal position of the scene, the difference between congruent and incongruent stimuli disappeared after the Synchronous visuo-tactile stimulation condition, but the effect due to congruent and incongruent for psychological distance words did not completely reverse (Proximal Words $=489 \mathrm{~ms}, 95 \%$ CI $=[456,522]$, Distal Words $=505 \mathrm{~ms}, 95 \% \mathrm{CI}=[476,533], \mathrm{p}=0.07)$, as seen for words presented in the distal position. This result suggests that, although affected by the FBI, participants did not perceive themselves fully disembodied from their physical body (see also Lenggenhager et al., 2007).

For the Asynchronous stimulation condition, Newman Keuls post-hoc test confirmed that participants judged the spatial distance of words expressing "psychological distance" faster when the words' semantic meaning matched the spatial distance in the scene from the participant's body, both for the distal and proximal position; this suggests that there was no change in semantic processing in the asynchronous control condition (Distal position: Distal Words $=506 \mathrm{~ms}, 95 \% \mathrm{CI}=[470,542]$, Proximal Words $=527 \mathrm{~ms}, 95 \% \mathrm{CI}=[489,564], \mathrm{p}<0.03$; Proximal position: Distal Words $=524$ $\mathrm{ms}, 95 \% \mathrm{CI}=[491,557]$, Proximal Words $=497 \mathrm{~ms}, 95 \% \mathrm{CI}=[463,531], \mathrm{p}<0.01)$.

We also run a second ANOVA for Control blocks taking into account the spatial location of the words in the image. Data were entered in a repeated measure ANOVA with Stimulation (Synchronous, Asynchronous), Spatial Location (Far, Near), and Words ("Screw", "Nail”) as within subject factors, and Experiment (Experiment 1, Experiment 2) as between subject factor. We found a main effect of Spatial Location 
$\left[\mathrm{F}(1,22)=5.15, \mathrm{p}<0.04, \eta^{2}=0.19\right]$, showing that participants were generally faster in responding to spatially proximal $(496 \mathrm{~ms}, 95 \% \mathrm{CI}=[474,518])$ then distal words (507 ms, 95\% CI $=[484,530]$, as in Bar-Anan et al., 2007). This effect was not modulated by Words or Stimulation, as any interaction of Spatial Location with other factors was not significant (all $\mathrm{p}_{\mathrm{s}}>0.08$ ). No other main effect was significant $\left(p_{s}>0.31\right.$; See Table 2 and 3 for detailed results for Experiment 1 and Experiment 2). We then looked at the relationship between the strength of the illusion, as assessed through the questionnaire, and the strength of the effect on the implicit association task. We calculated an index of the effect of the illusion by subtracting the Asynchronous score to the Synchronous one for each set of questions (Experimental and Control, separately) of the FBI questionnaire. A positive Illusion Index (II) indicates an effect of FBI in modulating BSC in the Synchronous condition, while a negative one indicates no effect of the FBI.

For the implicit association task, we calculated an index of the congruency effect (Incongruent - Congruent). Such Congruency Index (CI) was calculated for Distal and Proximal condition separately, both for Synchronous and Asynchronous conditions. A positive value indicates a classical congruency effect (i.e. participants are faster in responding to a congruent trial than to an incongruent one), while a negative value indicates no congruency effect.

Our hypothesis suggested that, if the change we observed in the implicit association task is related to a dissociation between the experienced self and the physical body, the more participants experienced a change in BSC during the FBI, the more they should show a reduction of the classical congruency effects as assessed through the implicit association task, particularly for trials presented in the distal portion of space. We ran a first correlation analysis between the II obtained for the Experimental 
questions (average of Q1 to Q4) and the CI calculated for Psychological Distance blocks, taking into account the spatial location of the word in the scene (Near, Far) in the Synchronous condition. Results revealed no significant correlation (all $\mathrm{p}_{\mathrm{s}}>0.26$ ). Given our hypothesis, we then run a correlation analysis between two relevant questions for the aim of this study, - that is Q3 ("I felt as if the virtual body was my body") and Q4 ("I felt as if my body was drifting towards the virtual body") with the implicit association task Index, calculated for Psychological Distance blocks, taking into account the spatial location of the word in the scene (Proximal, Distal) in the Synchronous condition. Results revealed a negative correlation for Q4 only ( $r=-0.40$, $\mathrm{p}<0.05)$. Specifically, subjects that exhibited a stronger agreement at this question that is a perceived change in the location where they perceived the self to be located as towards the virtual avatar after a Synchronous stimulation - also showed a stronger reduction of the classical congruency effect for words presented in the distal position of the image. Put in a different way, the more participants perceived themselves to be at the avatar's position, the more they showed faster RTs when a word expressing either spatial (Experiment 1) or social (Experiment 2) proximity was presented in the distal position in the image, that is at the position of the avatar where participants perceived to be located after a Synchronous stimulation. 
a)

Distal position

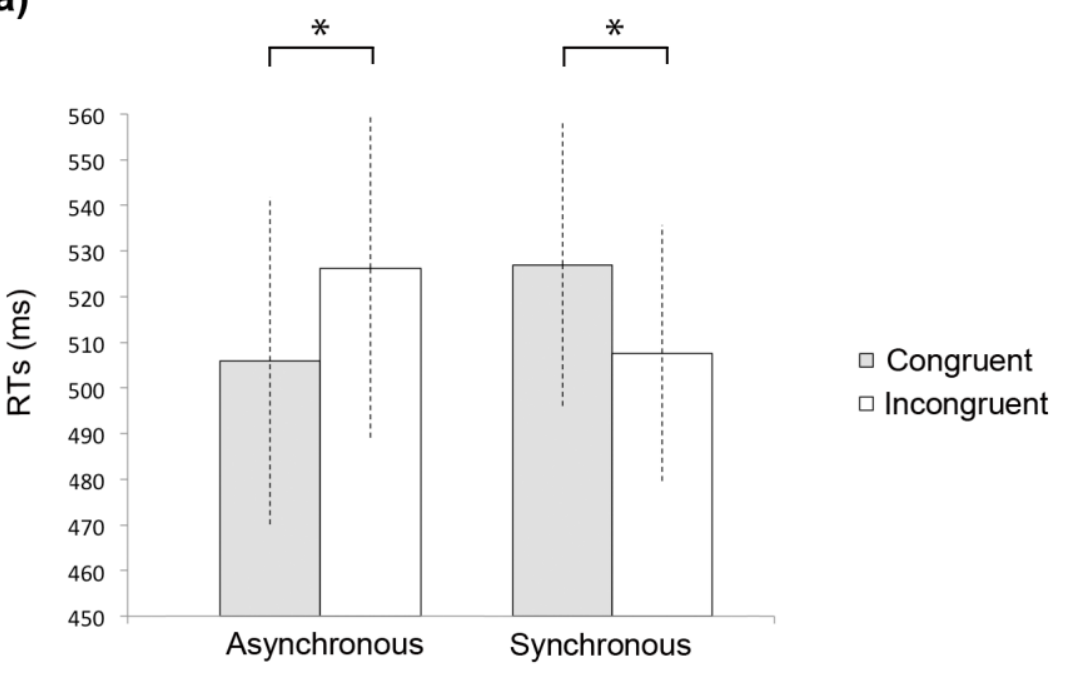

b)

Proximal position

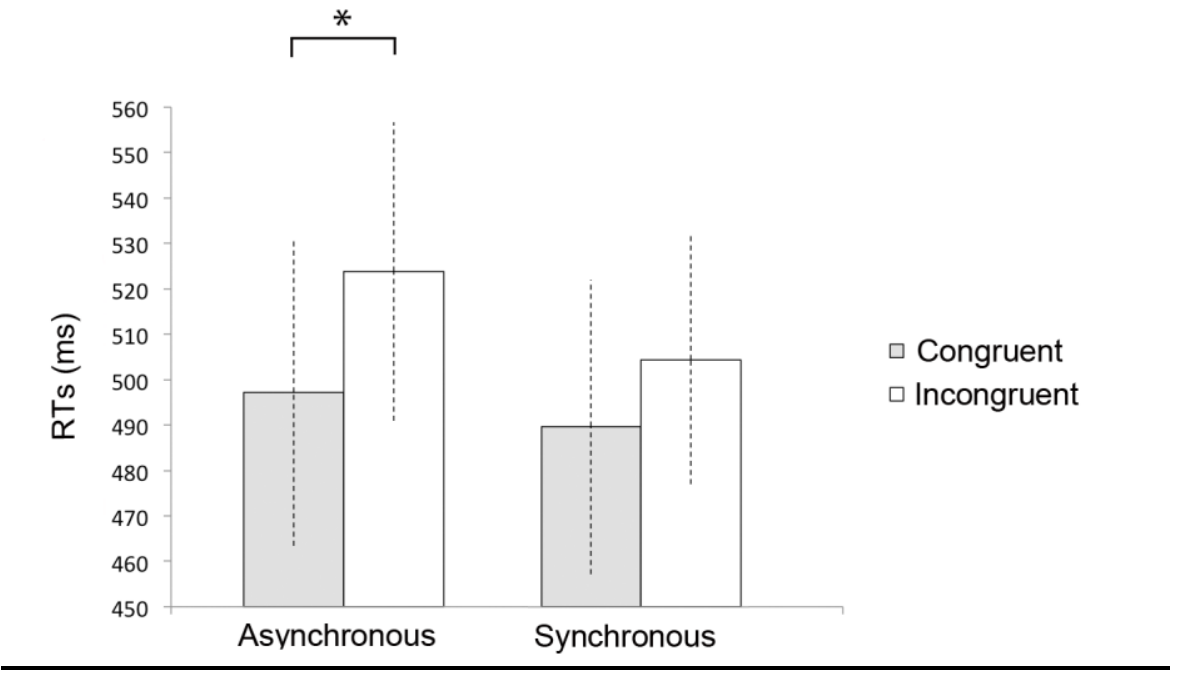

Figure 4. Implicit association task results: Proximal and Distal position.

The graph shows mean RTs for Distal (Panel A) and Proximal (Panel B) position of the words on the scene, for Synchronous and Asynchronous conditions. Congruent condition refers to trials where spatial location and psychological distance of the words matched (e.g. the word "Near" or "Us" presented at the proximal location in the image), while Incongruent refers to trials where spatial location and psychological distance of the words did not match (e.g. the word "Near" or "Us" presented at the distal position in the image). 


\begin{tabular}{|c|c|c|c|c|c|c|}
\hline & \multicolumn{3}{|c|}{ SYNCHRONOUS } & \multicolumn{3}{|c|}{ ASYNCHRONOUS } \\
\hline & $\begin{array}{l}\text { "Near" } \\
\text { Cong }\end{array}$ & $\begin{array}{l}\text { "Far" } \\
\text { Incong }\end{array}$ & $\begin{array}{l}\text { Congruency } \\
\text { Index (CI) }\end{array}$ & $\begin{array}{l}\text { "Near" } \\
\text { Cong }\end{array}$ & $\begin{array}{l}\text { "Far" } \\
\text { Incong }\end{array}$ & $\begin{array}{l}\text { Congruency } \\
\text { Index (CI) }\end{array}$ \\
\hline $\begin{array}{l}\text { Spatially } \\
\text { Proximal }\end{array}$ & $\begin{array}{l}483 \mathrm{~ms}, \mathrm{CI} \\
{[431,534]}\end{array}$ & $\begin{array}{l}501 \mathrm{~ms}, \mathrm{CI} \\
{[451,552]}\end{array}$ & $18 \mathrm{~ms}$ & $\begin{array}{l}474 \mathrm{~ms}, \mathrm{CI} \\
{[435,513]}\end{array}$ & $\begin{array}{l}500 \mathrm{~ms}, \mathrm{CI} \\
{[454,546]}\end{array}$ & $26 \mathrm{~ms}$ \\
\hline & $\begin{array}{l}\text { "Near" } \\
\text { Incong }\end{array}$ & $\begin{array}{l}\text { "Far" } \\
\text { Cong }\end{array}$ & & $\begin{array}{l}\text { "Near" } \\
\text { Incong }\end{array}$ & $\begin{array}{l}\text { "Far" } \\
\text { Cong }\end{array}$ & \\
\hline $\begin{array}{c}\text { Spatially } \\
\text { Distal }\end{array}$ & $\begin{array}{l}501 \mathrm{~ms}, \mathrm{CI} \\
{[463,537]}\end{array}$ & $\begin{array}{l}518 \mathrm{~ms}, \mathrm{CI} \\
{[472,564]}\end{array}$ & $-18 \mathrm{~ms}$ & $\begin{array}{l}516 \mathrm{~ms}, \mathrm{CI} \\
{[460,571]}\end{array}$ & $\begin{array}{l}489 \mathrm{~ms}, \mathrm{CI} \\
{[441,536]}\end{array}$ & $28 \mathrm{~ms}$ \\
\hline & "Screw" & "Nail" & & "Screw" & “Nail" & \\
\hline $\begin{array}{l}\text { Spatially } \\
\text { Proximal }\end{array}$ & $\begin{array}{l}488 \mathrm{~ms}, \mathrm{CI} \\
{[450,527]}\end{array}$ & $\begin{array}{l}481 \mathrm{~ms}, \mathrm{CI} \\
{[445,513]}\end{array}$ & -- & $\begin{array}{l}484 \mathrm{~ms}, \mathrm{CI} \\
{[441,527]}\end{array}$ & $\begin{array}{l}498 \mathrm{~ms}, \mathrm{CI} \\
{[454,540]}\end{array}$ & --- \\
\hline $\begin{array}{c}\text { Spatially } \\
\text { Distal }\end{array}$ & $\begin{array}{l}499 \mathrm{~ms}, \mathrm{CI} \\
{[458,541]}\end{array}$ & $\begin{array}{l}509 \mathrm{~ms}, \mathrm{CI} \\
{[456,562]}\end{array}$ & -- & $\begin{array}{l}487 \mathrm{~ms}, \mathrm{CI} \\
{[454,519]}\end{array}$ & $\begin{array}{l}492 \mathrm{~ms}, \mathrm{CI} \\
{[455,528]}\end{array}$ & --- \\
\hline
\end{tabular}

Table 2. Experiment 1. Mean RTs with 95\% confidence intervals, shown separately for each word in Distal and Proximal location of the image, both for Synchronous (on the left) and Asynchronous (on the right) stimulation. The Congruency Index (CI) was calculated for Distal and Proximal condition separately, both for Synchronous and Asynchronous conditions, for Psychological Distance words only. A positive value indicates a classical congruency effect (i.e. participants are faster in responding to a congruent trial than to an incongruent one), while a negative value indicates no congruency effect. 
compared by means of a repeated measure ANOVA with Stimulation (Synchronous, Asynchronous) and Congruency (Congruent, Incongruent) as within-subjects factors. RTs from blocks with control words were instead analysed by means of a separate repeated measure ANOVA with Stimulation (Synchronous, Asynchronous) and Words ("Screw", "Nail") as within subject factors.

For psychological distance blocks, results revealed a significant Congruency effect $\left[F(1,11)=12.06, p<0.01, \eta^{2}=0.52\right]$. As clearly shown in Figure 5, Participants were significantly faster with congruent trials (Mean, 95\% confidence intervals or CI: $438 \mathrm{~ms}, 95 \% \mathrm{CI}=[403,473]$ as compared to incongruent trials $(450 \mathrm{~ms}, 95 \% \mathrm{CI}=$ $[412,488]$. Such an effect was independent from the visuo-tactile stimulation, as the interaction with the factor Stimulation, as well as the main factor Stimulation itself, were not significant (all $\left.\mathrm{p}_{\mathrm{s}}>0.75\right)$.

For Control blocks, in line with the previous experiments, RTs were neither affected by Stimulation nor Words, as no main effect of Stimulation $[F(1,11)=0.07, p=0.80$, $\left.\eta^{2}=0.006\right]$ or Words $\left[F(1,11)=0.07, p=0.80, \eta^{2}=0.05\right]$, nor the two-way interaction were significant $\left.\left[F(1,11)=0.26, p=0.62, \eta^{2}=0.02\right]\right)$. 


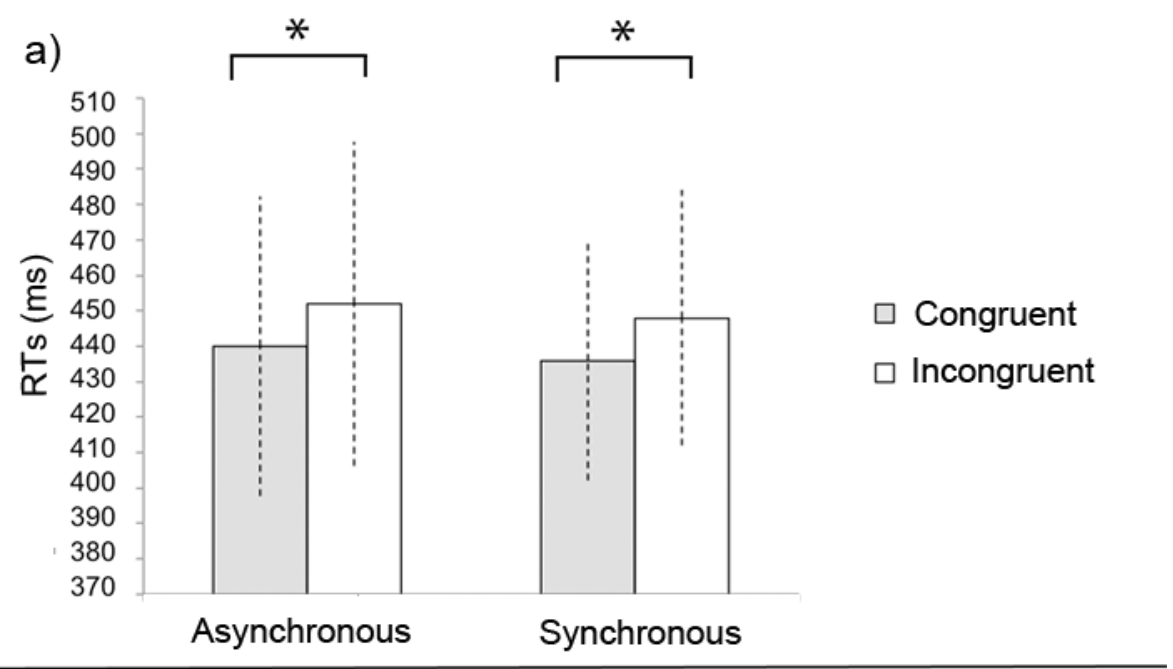

b)

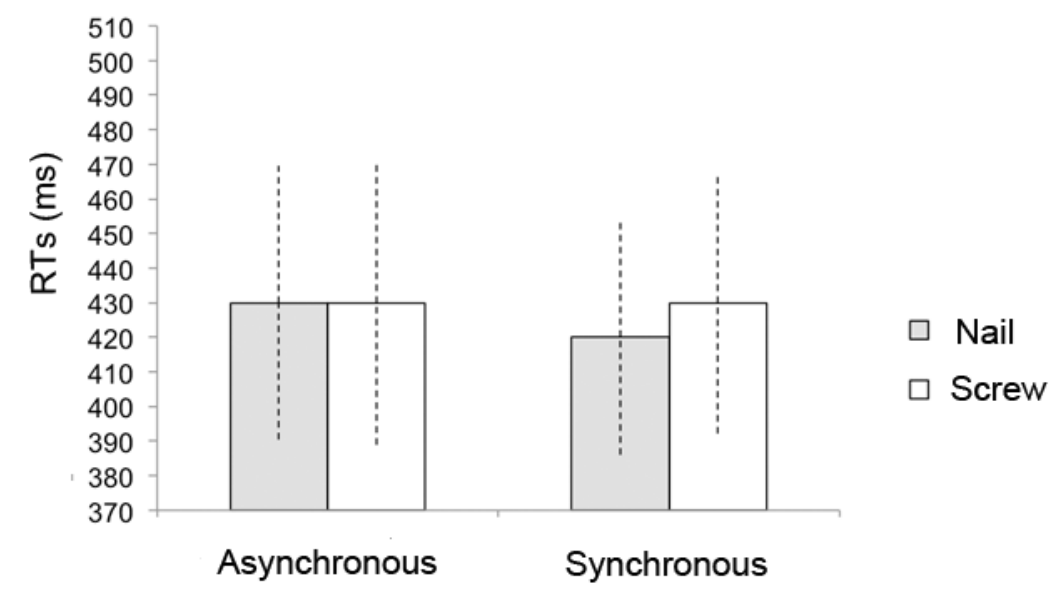

Figure 5. Experiment 3. Implicit Association Task results. Mean RTs for target words shown separately for Psychological Distance (Panel A) and Control (Panel B) blocks.

Panel A. Mean RTs for Congruent and Incongruent conditions both for Synchronous and Asynchronous stimulation. Congruent condition refers to trials where spatial location matched the psychological distance of the words (e.g. the word "Near" presented at the proximal location in the image), while Incongruent refers to trials where spatial location and psychological distance of the words did not match (e.g. the word "Near" presented at the distal position in the image).

Panel B. Mean RTs for control Words (Screw, Nail) both for Synchronous and Asynchronous stimulation.

\section{Discussion}

In the present study we show that altering the spatial unity between the physical body and the self by means of conflicting multisensory stimulation affects cognitive 
processing of abstract concepts conveyed by words. The CLT implies that different concepts (spatial, temporal, social, and probabilistic) are automatically computed in terms of "psychological distance" from a point of origin coinciding with the physical body (Liberman \& Trope, 2008; 2014). Here, we manipulated multisensory stimulation in order to induce the FBI, thus producing a dissociation between the experienced self and the physical body, so that participants self-identified with a virtual body placed far apart. Under such circumstances, we found that the point of origin to which "psychological distances" are referenced shifts from the location of the physical body to the location of the experienced self. Indeed, when participants identified with the avatar placed at the far location in the scene, due to the FBI manipulation, the congruency effect between semantic and physical distance of words presented in the scene was abolished as compared to a control condition of asynchronous visuo-tactile stimulation. More specifically, this effect was due to a change in processing the words located at the distal location of the scene: during the FBI words conveying proximal psychological distance - both in the spatial domain, i.e. "Near", and in the social domain, i.e. "Us" - induced faster reaction times when presented at the distal position (i.e. in Incongruent condition, see Figure 4), corresponding to the position where the avatar was located and towards where the experienced self shifted as compared to words conveying distal psychological distance. On the contrary, we did not observe faster RTs for words conveying distal psychological distance (both in the spatial domain, i.e. "Far", and in the social domain, i.e. "Them") when these were presented at the proximal position of the scene (that is, in a position now far from the experienced self in the synchronous condition as induced by the FBI). Such a result suggests that the concept of closeness to the physical body was not completely reversed even when the FBI was successfully 
induced. We can speculate that although affected by the FBI, participants did not perceive themselves fully disembodied from their physical body. Indeed, it has been already demonstrated that, even if the FBI is able to induce a sense of ownership over another virtual body or body part, healthy participants do not achieve a complete disconnection with their own body. In this view, we can speculate that participants always partially refer to proximal words presented in the proximal space as slightly congruent, even if the effect is reduced as compared to a condition of asynchronous stimulation (i.e. where no embodiment over the avatar was present). It has been proposed that the illusory ownership process described after FBI could be the results of stroking-induced changes in the size and position of trunk-centred bimodal neurons with respect to the virtual body (Blanke, 2012). These multisensory neurons, who have been described at the level of premotor and parietal cortices, integrate somatosensory, visual and/or auditory signals related to specific body parts (Graziano Cooke, \& Taylor, 2000; Graziano, Reiss, \& Gross, 1999) or even the whole body both in monkeys and human. Since the FBI is achieved as a consequence of a synchronous visuo-tactile stroking at the back while seeing the avatar being stroked at the same time, it has been hypothesized that the visual receptive fields of such neurons would be enlarged following visuo-tactile stroking. Such enlargement does not reflect that the visual receptive field of these neurons does not respond anymore to the stimuli presented at the body, but that, as a consequence of the synchronous visuo-tactile stroking, they integrated also visual stimuli presented at the virtual body.

Such result fits well also with previous data (see Holmes, Calvert \& Spence, 2004) on modulation of visuo-tactile interactions due to tool-use (assessed through the cross modal congruency task). Active tool-use increased the salience or effectiveness of visual stimuli presented at the tip(s) of the hand-held tool(s), but revealed the 
persistence of visuo-tactile interactions at the body (that is at the hand/handle level). Said in a different way, following tool-use, the multisensory peri-hand area where visual and tactile stimuli are normally integrated is pushed farther in space up to where the goal of the action is. However, it has been shown that some forms of multisensory interactions remain at the body level, suggesting that even if body and space representations are extended up to the far space, multisensory interactions that normally takes place around the body does not disappear.

Several studies have previously demonstrated that changes in BSC as those induced during the FBI affect processing of low-level information. For instance, embodying an avatar has been proved to affect how people visually perceive external objects in the world. Previous studies indeed showed that inducing illusory ownership for a virtual body that is bigger or smaller than the participants' body made participants perceive objects to be respectively smaller and bigger or to be placed at a closer or a farther distance (Linkenauger, Ramenzoni, \& Proffitt, 2010; Banakou et al., 2013; van der Hoort \& Ehrsson, 2014). Moreover, it is also known that a change in BSC as induced by the FBI affects low-level processing of body-related signals, such as visuo-tactile integration (Aspell et al., 2009), physiological reactions (Petkova \& Ehrsson, 2008; Slater et al., 2008), pain perception (Romano et al., 2014) and body temperature (Salomon et al., 2013).

Whereas these previous studies reported modifications of low-level sensory processing due to changes in BSC, a different line of work (Coventry, Valdés, Castillo, \& Guijarro-Fuentes, 2008) proposed an interesting link between a low-level, sensory-motor manipulation, such as tool-use, and high-level linguistic processing. In this work, participants were asked to verbally indicate the position of different target objects placed in front of them, by using demonstrative adjectives, while pointing at 
them either with their hand or with a $70 \mathrm{~cm}$ stick. Using the stick increased the use of proximal demonstratives when indicating the position of far objects, such as objects were perceived as closer to the body because of the enhanced possibility of interacting with them through the tool. Tool-use represents a manipulation that, by physically linking the hand and the far space, extends the space where the body acts (Serino, Bassolino, Farnè, \& Làdavas, 2007; Canzoneri, Ubaldi, Rastelli, Finisguerra, Bassolino, \& Serino, 2013; Cardinali, Frassinetti, Brozzoli, Urquizar, Roy, \& Farnè, 2009). In the present study, however, we reveal a new link between body perception and cognitive functions, at a higher level of complexity, by showing that inducing an alteration in BSC with respect to a virtual body that is placed at a distant location by means of the FBI - and thus without altering the physical position of the body impacts on conceptual processing of words expressing different levels of psychological distance. In particular, our results indicate that when participants selfidentified with a virtual body placed at a spatial location that differs from that of their physical body, the reference frame the brain used to compute abstract concepts, which normally refers to the physical body, is shifted to the position of the avatar participants self-identified with. Indeed, while normally the processing of words expressing psychological proximity, e.g. "Near", is facilitated when presented close to the participant's body, this was different when the FBI was induced: now we found a facilitation effect of comparable size when psychologically proximal words were presented at a far location and thus in "proximity" of the avatar participants selfidentified with. Interestingly, the fact that this effect was found for proximal words presented in the distal position only (and not for distal words presented in a proximal position) seems to rule out the possibility that the effect described in this study is due to a general distracting effect due to multisensory conflict induced in the synchronous 
condition only. Rather, this result supports the idea that the congruency effects previously described in the CLT depends on the encoding of the reference point of psychological distance not only at the location of the physical body, but also at that of the experienced self.

Interestingly, the same effects were obtained for words expressing psychological distance in the spatial domain ("Near" and "Far", Experiment 1) and the social domain ("Us" and "Them", Experiment 2). These results suggest that interfering with the spatial unity between the physical body and the experienced self affects the processing not only of spatial concepts, but also of more abstract social concepts, which, accordingly to the CLT, are computed in terms of psychological distance (Liberman \& Trope, 2008; 2014). By definition, "psychological distance" is an egocentric construct, in the sense that is frequently conceived as the distance from the body (see Trope \& Liberman, 2010, for a review) across different dimensions. An event is more psychologically distant as it occurs in more remote locations, or as it takes place farther into the future or the past, or as it concerns people we perceive as being different from us. All previous work, however, did not focus between the distance from the self and the distance from the body, as these normally coincide. The novelty of our results is that, by experimentally manipulating through visuo-tactile stimulation the spatial unity between the body and the self, we demonstrated that psychological distance is not computed as strictly referenced to the location of physical body, but to that of the experienced self. Moreover, the CLT framework relates psychological distance to the dimension of concreteness and abstraction, by suggesting that individuals use concrete, low-level construals to represent proximal events, while they use more abstract, high-level construal to represent distal events, and this difference depends on the level of information available about a specific 
event. When something happens "here and now" (i.e. a more proximal event), information available is likely high and therefore we represent that event in concrete, more structured and low-level terms. Instead as an event is further removed from direct experience (i.e., more distant), less information is available, leading to the formation of a more abstract and high-level representation of the event. In order to manipulate the level of representation, previous studies in the field of CLT used topdown manipulations. For instance they asked people to imagine a scenario taking place either in the near (low-level condition) or far (high-level condition) future (see Trope, Liberman \& Wakslak, 2007, for a review) in order to prime respectively a low - or a high-level construal, and showed an effect of such a manipulation in an implicit association task. More generally, Maglio, Trope and Liberman (2013) demonstrated that experiencing any kind of psychological distance reduces sensitivity to other distances. On a related aspect, Maglio and Trope (2012) also demonstrated that manipulating the level of a construal, that is priming participants towards a more concrete (low-level) or abstract (high-level) aspects of an activity (top-down manipulation), affected contextual body information and this was reflected in a different performance in a task of visual length estimate and on importance ratings. These results are complimentary to our study, that, instead, shows that a bottom-up manipulation, based on controlled forms of visuo-tactile stimulation, that allows experimentally inducing a "physical" distance between the self and the body, affects the strength of the processing of psychological distance-related concepts, thus impacting on high-level processing within the CLT field.

It is worth noting, however, that the concepts or physical and social distances are both more or less directly tied to the self, while additional dimensions of psychological distance, such as temporal distance or probability, are only indirectly related to the 
self. It would be interesting to investigate whether any change in the relationship between the body and the self would affect the congruency effect also for different dimensions for psychological distance. Previous studies in the field of the CLT demonstrated that the different dimensions of psychological distances have an inherent common meaning and the activation of one distance dimension automatically activate the other dimensions (see e.g., Bar-Anan et al., 2007 and Trope \& Liberman, 2010 for a review). So, in principle, if we replicate exactly the same experiment by using the temporal or probability distance words, we should find the same effect. Future studies are needed to further investigate this issue.

More generally, the present data are related to some recent accounts proposing that the human mind has developed a common system to project self-representations along different dimensions. Mesulam (2001) referred to self-projection as transposing "the effective reference point of perception from self to other, from here to there, and from now to then". It has been proposed that the ability of traversing psychological distance, thinking about the future, remembering the past, and taking others' perspective are all features of the human mind that require a common ability of abstracting the self from the "here and now" of its physical body (Schacter, Addis, \& Buckner, 2007; Buckner \& Carroll, 2007; Arzy et al., 2008; see also Molnar-Szakacs \& Arzy, 2009 for a review).

Recently, Bergouignan and colleagues (Bergouignan, Nyberg, \& Ehrsson, 2014) demonstrated the necessary role of perceiving the world from one's own body perspective for the successful encoding of episodic memories, both at behavioural and neuroimaging level. This study suggests a link between the experience of the self and memory encoding: when a distance is introduced between the perceived and the physical self, the default egocentric information processing at memory level is 
affected. Our findings are in line with Bergouignan's results by showing that multisensory body self-perception directly influences a specific higher cognitive function, namely the processing of distance related semantic information.

Here we provide an empirical demonstration that if the spatial unity between the self and physical body is altered by means of a low-level manipulation of multisensory body inputs (i.e., synchronous visuo-tactile stimulation), the processing of abstract concepts implying different psychological distances is congruently and predictably affected. 


\section{Author contributions}

AS, EC and GdP conceived the study. EC and $\mathrm{BH}$ realized the paradigm. EC performed the experiments. EC and AS analysed the data and drafted the manuscript. GdP, $\mathrm{BH}, \mathrm{OB}$ provided critical revisions. AS, BH and OB supervised the project. All authors approved the final version of the manuscript for submission.

\section{Acknowledgements}

Support for this work was provided by W Investments S.A., Switzerland (industrial grant 'RealiSM').

The authors thank Roberta Ronchi, Nathan Faivre and Roy Salomon for their useful comments and suggestions on data analysis and interpretation, and Nadia Berard and Simone Bonaccorsi for their help in data collection. 


\section{REFERENCES}

Arzy, S., Molnar-Szakacs, I., \& Blanke, O. (2008). Self in time: imagined selflocation influences neural activity related to mental time travel. The Journal of Neuroscience: The Official Journal of the Society for Neuroscience, 28(25), 6502-6507. http://doi.org/10.1523/JNEUROSCI.5712-07.2008

Aspell, J. E., Heydrich, L., Marillier, G., Lavanchy, T., Herbelin, B., \& Blanke, O. (2013). Turning body and self inside out: visualized heartbeats alter bodily self-consciousness and tactile perception. Psychological Science, 24(12), 2445-2453. doi:10.1177/0956797613498395

Aspell, J. E., Lavanchy, T., Lenggenhager, B., \& Blanke, O. (2010). Seeing the body modulates audiotactile integration. The European Journal of Neuroscience, 31(10), 1868-1873. doi:10.1111/j.1460-9568.2010.07210.x

Aspell, J. E., Lenggenhager, B., \& Blanke, O. (2009). Keeping in touch with one's self: multisensory mechanisms of self-consciousness. PloS One, 4(8), e6488. doi:10.1371/journal.pone.0006488

Banakou, D., Groten, R., \& Slater, M. (2013). Illusory ownership of a virtual child body causes overestimation of object sizes and implicit attitude changes. Proceedings of National Academies of Science USA, 110(31), 12846-51. doi: 10.1073/pnas. 1306779110

Bar-Anan, Y., Liberman, N., Trope, Y., \& Algom D. (2007). Automatic processing of psychological distance: evidence from a Stroop task. Journal of Experimental Psychology, 136(4), 610-22.

Barsalou, L. W. (2008). Grounded cognition. Annual Review of Psychology, 59, 617645. doi:10.1146/annurev.psych.59.103006.093639 
Bergouignan, L., Nyberg, L., \& Ehrsson, H. H. (2014). Out-of-body-induced hippocampal amnesia. Proceedings of the National Academy of Sciences of the United States of America, 111, 4421-6.

Blanke, O. (2012). Multisensory brain mechanisms of bodily self-consciousness. Nature Reviews. Neuroscience, 13(8), 556-571. doi:10.1038/nrn3292

Blanke, O., \& Metzinger, T. (2009). Full-body illusions and minimal phenomenal selfhood. Trends in Cognitive Sciences, 13(1), 7-13. doi:10.1016/j.tics.2008.10.003

Blanke, O., Slater, M., Serino, A. (2015). Behavioral, Neural, and Computational Principles of Bodily Self-Consciousness. Neuron; 88(1):145-66. doi: 10.1016/j.neuron.2015.09.029.

Borghi AM, Cimatti F (2010) Embodied cognition and beyond: acting and sensing the body. Neuropsychologia 48: 763-773.

Broughton, R., Wasel, N. A. (1990) Text-stimuli presentation manager for the IBM PC with ipsatization correction for response sets and reaction times. Behavioral Research Method Ins C, 22(4):421-3.

Buckner, R. L., \& Carroll, D. C. (2007). Self-projection and the brain. Trends in Cognitive Sciences, 11(2), 49-57. doi:10.1016/j.tics.2006.11.004

Canzoneri, E., Ubaldi, S., Rastelli, V., Finisguerra, A., Bassolino, M., \& Serino, A. (2013). Tool-use reshapes the boundaries of body and peripersonal space representations. Experimental Brain Research, 228(1), 25-42. doi:10.1007/s00221-013-3532-2.

Cardinali, L., Frassinetti, F., Brozzoli, C., Urquizar, C., Roy, A. C., \& Farnè, A. (2009). Tool-use induces morphological updating of the body schema. Current Biology: CB, 19(12), R478-479. doi:10.1016/j.cub.2009.05.009 
Cattell, R.B. Psychological measurement: ipsative, normative and interactive. Psychol Rev 1944; 51:292-303.

Coventry, K. R., Valdés, B., Castillo, A., \& Guijarro-Fuentes, P. (2008). Language within your reach: near-far perceptual space and spatial demonstratives. Cognition, 108(3), 889-895. doi:10.1016/j.cognition.2008.06.010

Ehrsson, H. H. (2007). The experimental induction of out-of-body experiences. Science (New York, N.Y.), 317(5841), 1048. doi:10.1126/science.1142175

Gallagher, S. (2005). How the body shapes the mind. New York, Oxford University Press, 284 pp ISBN: 284, 0199271941.

Gibbs, R.W. (2006). Embodiment and Cognitive Science. New York: Cambridge Univ. Press.

Graziano, M.S., Cooke, D.F., \& Taylor, C.S. (2000). Coding the location of the arm by sight. Science, 290(5497), 1782-1786.

Graziano, M.S., Reiss, L.A., Gross, C.G. (1999). A neuronal representation of the location of nearby sounds. Nature, 397(6718), 428-430.

Guterstam, A, Abdulkarim, Z., Ehrsson, H.H. (2015) Illusory ownership of an invisible body reduces autonomic and subjective social anxiety responses. Sci Rep. 5:9831. doi: 10.1038/srep09831.

Hänsel, A., Lenggenhager, B., von Känel, R., Curatolo, M., \& Blanke, O. (2011). Seeing and identifying with a virtual body decreases pain perception. European Journal of Pain (London, England), 15(8), 874-879. doi:10.1016/j.ejpain.2011.03.013

Holmes, N. P., Calvert, G. A., \& Spence, C. (2004). Extending or projecting peripersonal space with tools? Multisensory interactions highlight only the distal and proximal ends of tools. Neuroscience Letters, 372(1-2), 62-67. 
http://doi.org/10.1016/j.neulet.2004.09.024

Lenggenhager, B., Tadi, T., Metzinger, T., \& Blanke, O. (2007). Video ergo sum: manipulating bodily self-consciousness. Science (New York, N.Y.), 317(5841), 1096-1099. doi:10.1126/science.1143439.

Liberman, N., \& Trope, Y. (2008). The psychology of transcending the here and now. Science (New York, N.Y.), 322(5905), 1201-1205. doi:10.1126/science.1161958.

Liberman, N., \& Trope, Y. (2014). Traversing psychological distance. Trends in Cognitive Sciences, 18(7), 364-369. doi:10.1016/j.tics.2014.03.001

Linkenauger, S.A., Ramenzoni, V., \& Proffitt, D.R. (2010). Illusory shrinkage and growth: body-based rescaling affects the perception of size. Psychological Science, 21(9), 1318-25. doi: 10.1177/0956797610380700.

Maglio, S.J., Trope, Y., Liberman, N. (2013) Distance from a distance: psychological distance reduces sensitivity to any further psychological distance. J Exp Psychol Gen; 142(3):644-57. doi: 10.1037/a0030258.

Maglio, S.J., Trope, Y. (2012) Disembodiment: abstract construal attenuates the influence of contextual bodily state in judgment. J Exp Psychol Gen.; 141(2):211-6. doi: 10.1037/a 0024520.

Maister, L., Sebanz, N., Knoblich, G., Tsakiris, M. (2013). Experiencing ownership over a dark-skinned body reduces implicit racial bias. Cognition, 128(2):170-8. doi: 10.1016/j.cognition.2013.04.002.

Maister, L., Slater, M., Sanchez-Vives, M.V., Tsakiris, M. (2015). Changing bodies changes minds: owning another body affects social cognition. Trends Cogn Sci, 19(1):6-12. doi: 10.1016/j.tics.2014.11.001.

Mesulam, M. The human frontal lobes: Transcending the default mode through 
contingent encoding. In: Stuss D, Knight R, (eds). Principles of Frontal Lobe Function. Oxford: Oxford University Press 2002; 8-30.

Molnar-Szakacs, I., \& Arzy, S. (2009). Searching for an integrated self-representation. Communicative \& Integrative Biology, 2(4), 365-367.

Moseley, G. L., Olthof, N., Venema, A., Don, S., Wijers, M., Gallace, A., \& Spence, C. (2008). Psychologically induced cooling of a specific body part caused by the illusory ownership of an artificial counterpart. Proceedings of the National Academy of Sciences of the United States of America, 105(35), 13169-13173. doi:10.1073/pnas.0803768105

Peck, T.C., Seinfeld, S., Aglioti, S.M., Slater, M. (2013) Putting yourself in the skin of a black avatar reduces implicit racial bias. Conscious Cogn. 22(3):779-87. doi: 10.1016/j.concog.2013.04.016.

Petkova, V. I., \& Ehrsson, H. H. (2008). If I were you: perceptual illusion of body swapping. PloS One, 3(12), e3832. doi:10.1371/journal.pone.0003832

Pfeiffer, C., Lopez, C., Schmutz, V., Duenas, J. A., Martuzzi, R., \& Blanke, O. (2013). Multisensory origin of the subjective first-person perspective: visual, tactile, and vestibular mechanisms. PloS One, 8(4), e61751. doi:10.1371/journal.pone.0061751

Preston, C., \& Ehrsson, H.H. (2014). Illusory changes in body size modulate body satisfaction in a way that is related to non-clinical eating disorder psychopathology. PLoS One, 9(1):e85773. doi: 10.1371/journal.pone.0085773.

Romano, D., Pfeiffer, C., Maravita, A., \& Blanke, O. (2014). Illusory selfidentification with an avatar reduces arousal responses to painful stimuli. Behavioral Brain Research, 261:275-81. doi: 10.1016/j.bbr.2013.12.049.

Salomon, R., Lim, M., Pfeiffer, C., Gassert, R., \& Blanke O. (2013). Full body 
illusion is associated with widespread skin temperature reduction. Frontiers in Behavioral Neuroscience, 25; 7:65. doi: 10.3389/fnbeh.2013.00065.

Schacter, D. L., Addis, D. R., \& Buckner, R. L. (2007). Remembering the past to imagine the future: the prospective brain. Nature Reviews. Neuroscience, $8(9)$, 657-661. doi:10.1038/nrn2213

Serino, A., Bassolino, M., Farnè, A., Làdavas, E. (2007). Extended multisensory space in blind cane users. Psychological Science, 18(7):642-8.

Slater, M., Perez-Marcos, D., Ehrsson, H. H., \& Sanchez-Vives, M. V. (2008). Towards a digital body: the virtual arm illusion. Frontiers in Human Neuroscience, 2, 6. doi:10.3389/neuro.09.006.2008.

Slater, M, Spanlang, B., Sanchez-Vives, M.V., Blanke O. (2010). First person experience of body transfer in virtual reality. PLoS One; 5(5): e10564. doi: 10.1371/journal.pone.0010564.

Trope, Y., \& Liberman, N. (2010). Construal-Level Theory of Psychological Distance. Psychological Review, 117(2), 440-463. doi:10.1037/a0018963.

Trope, Y., Liberman, N., \& Wakslak, C. (2007). Construal Levels and Psychological Distance: Effects on Representation, Prediction, Evaluation, and Behavior. Journal of Consumer Psychology: The Official Journal of the Society for Consumer Psychology, 17(2), 83-95. http://doi.org/10.1016/S10577408(07)70013-X.

Tsakiris, M. (2010) My body in the brain: a neurocognitive model of body-ownership. Neuropsychologia;48(3):703-12.doi: 10.1016/j.neuropsychologia.2009.09.034. van der Hoort, B., \& Ehrsson, H.H. (2014). Body ownership affects visual perception of object size by rescaling the visual representation of external space. Atten Percept Psychophys, 76(5):1414-28. doi: 10.3758/s13414-014-0664-9. 
Vogeley, K., \& Fink, G. R. (2003). Neural correlates of the first-person-perspective. Trends in Cognitive Sciences, 7(1), 38-42. 